\title{
Optimal protocols for quantum quenches of finite duration in the Luttinger model
}

\author{
Ádám Bácsi,,$^{1,2, *}$ Masudul Haque, ${ }^{3,4}$ and Balázs Dóra ${ }^{1,5}$ \\ ${ }^{1}$ MTA-BME Lendület Topology and Correlation Research Group, Budapest University of Technology and Economics, 1521 Budapest, Hungary \\ ${ }^{2}$ Department of Mathematics and Computational Sciences, Széchenyi István University, 9026 Györ, Hungary \\ ${ }^{3}$ Department of Theoretical Physics, Maynooth University, County Kildare, Ireland \\ ${ }^{4}$ Max Planck Institute for the Physics of Complex Systems, Nöthnitzer Strasse 38, 01187 Dresden, Germany \\ ${ }^{5}$ Department of Theoretical Physics, Budapest University of Technology and Economics, 1521 Budapest, Hungary
}

(Received 6 March 2019; published 6 June 2019)

\begin{abstract}
Reaching a target quantum state from an initial state within a finite temporal window is a challenging problem due to nonadiabaticity. We study the optimal protocol for switching on interactions to reach the ground state of a weakly interacting Luttinger liquid within a finite time $\tau$, starting from the noninteracting ground state. The protocol is optimized by minimizing the excess energy at the end of the quench, or by maximizing the overlap with the interacting ground state. We find that the optimal protocol is symmetric with respect to $\tau / 2$, and can be expressed as a functional of the occupation numbers of the bosonic modes in the final state. For short quench durations, the optimal protocol exhibits fast oscillation and excites high-energy modes. In the limit of large $\tau$, minimizing energy requires a smooth protocol while maximizing overlap requires a linear quench protocol. In this limit, the minimal energy and maximal overlap are both universal functions of the system size and the duration of the protocol.
\end{abstract}

DOI: 10.1103/PhysRevB.99.245110

\section{INTRODUCTION}

Progress in quantum technologies relies on our ability to manipulate quantum states, in particular, interacting manycomponent quantum states. A key challenge is to engineer the transfer of a quantum system from one ground state to another, without excitations, in finite time. Such a transfer is guaranteed by the adiabatic theorem if the duration of parameter change is allowed to be infinite. When this is performed in finite time, this is often referred to as a "shortcut to adiabaticity." Such techniques are an obvious route to improving the viability of quantum annealing and adiabatic quantum computing algorithms [1-3], for which unwanted excitations are of serious concern.

The problem of optimizing a quantum quench of finite duration has been addressed in the context of a variety of quantum systems, including trapped particles or trapped BoseEinstein condensates [4-7], trapped interacting fermionic gases [8-10], Luttinger liquids [11], Majorana qubits [12,13], the Lipkin-Meshkov-Glick model [14-16], and spin systems [15,17-20]. Optimal protocols have been studied in quantum quenches through a quantum critical point $[14,19,21,22]$ and from a quantum critical point to the gapless phase of the Luttinger liquid [11].

In this paper, we consider the optimization of ramps of finite duration in a Luttinger liquid. Luttinger liquids appear as effective low-energy descriptions of gapless phases in various one-dimensional (1D) interacting systems [23-26]. For example, for fermions in one dimension, Landau's Fermiliquid description breaks down for any finite interaction- the

*bacsi.adam@sze.hu low-energy physics is described by bosonic collective modes with linear dispersion and is characterized by anomalous noninteger power-law dependences of correlation functions. The Luttinger model similarly arises as the low-energy description of spin chains or that of interacting 1D bosons [23-25]. In addition to its rich history in equilibrium condensed-matter physics, in the past dozen years the Luttinger model has also been used as a model system for nonequilibrium phenomena. Nonequilibrium studies using the Luttinger model include investigations of instantaneous quantum quenches [27-42], transport due to inhomogeneous initial conditions [43-49], and, most relevantly to the present paper, quenches of finite duration (finite rate) [50-57].

In the present paper, we consider quenches having a certain duration $\tau$, governed by a quench shape function $Q(t)$ such that $Q(0)=0$ and $Q(t>\tau)=1$. The system starts at $t=0$ in the ground state of the initial noninteracting Hamiltonian. To proceed analytically, we assume a weak final interaction, which allows for a perturbative, analytical treatment of the ensuing Bogoliubov equations. In general, for finite $\tau$ the final state after the quench differs from the ground state of the final Hamiltonian. The deviation can be quantified either by the excess energy of the final state relative to the target ground state, or by the overlap between the final state and the target ground state, i.e., the vacuum-to-vacuum probability. We consider both these measures, and find quench protocols $Q(t)$ that minimize the excess energy and those that maximize the vacuum-to-vacuum probability.

We first show that both the excess energy and the overlap depend only on the occupancies of bosonic modes at the end of the quench. We find that the derivative of the optimal protocol must be symmetric with respect to $\tau / 2$, and the protocol function itself must obey $Q(t)=1-Q(\tau-t)$. 
The shape of the quench of finite duration is parametrized as a Fourier series, and its coefficients are optimized. Fast protocols excite high-energy modes, and thus are nonuniversal in the Luttinger liquid sense. With increasing $\tau$, the excess energy is minimized by a smooth protocol while the overlap is maximized by a linear ramp. In this limit, the minimal energy and maximal overlap are both universal functions of the system size and the duration of the protocol.

In Sec. II, we first introduce the model, the quench protocol, and notations, and then derive expressions for the excess energy and for the overlap with the final ground state. The parity of the optimal quench protocol is considered in Sec. III. In Secs. IV and V we report on the optimization of $Q(t)$ by, respectively, minimizing the final energy and maximizing the final overlap with the target state. Section VI provides some concluding discussion.

\section{QUANTUM QUENCH IN THE LUTTINGER MODEL}

The low-energy behavior of a one-dimensional electron system is described by the Luttinger model. This model has the advantage that both the noninteracting and the interacting system can be diagonalized analytically. This is because both the kinetic and the interaction energy can be expressed as quadratic terms of bosonic creation and annihilation operators describing electron-hole excitations. The time-dependent Hamiltonian governing the quantum quench is given as

$$
H(t)=H_{0}+Q(t) V
$$

where

$$
H_{0}=\sum_{q>0} \omega_{0}(q)\left(b_{q}^{+} b_{q}+b_{-q}^{+} b_{-q}\right)
$$

is the Hamiltonian of the noninteracting system with $\omega_{0}(q)=v|q|$. In the formula $b_{q}$ is the bosonic annihilation operator corresponding to the wave number $q$. The second term in Eq. (1) describes the electron-electron interaction:

$$
V=\sum_{q>0} g(q)\left(b_{q}^{+} b_{-q}^{+}+b_{q} b_{-q}\right)
$$

where $g(q)=g_{2}|q| e^{-v \tau_{0}|q|}$. Note that in the interaction only backscattering $\left(g_{2}\right)$ is considered. It can be shown that the forward scattering $\left(g_{4}\right)$ does not effect the bosonic occupation numbers to leading order in the interaction strength and, hence, can be neglected. The time scale of $\tau_{0}$ is introduced to model the high-energy cutoff and is assumed to be inversely proportional to the bandwidth of the electron system.

In Eq. (1), $Q(t)$ describes the quench protocol with the duration of $\tau$, i.e.,

$$
Q(t)= \begin{cases}0 & \text { if } t<0 \\ Q(t) & \text { if } 0<t<\tau \\ 1 & \text { if } t>\tau\end{cases}
$$

where the nontrivial time dependence happens in the intermediate interval.

If the quench is adiabatic, i.e., in the $\tau \rightarrow \infty$ limit, the system is expected to arrive in the ground state of the interacting system after the quench and no bosonic excitations are present. However, if the quench duration is finite, the final state is presumably not the pure ground state of the interacting Hamiltonian but is a linear combination of the ground state and excited states.

The bosonic excitations of the interacting system are described by the operators of

$$
d_{ \pm q}=b_{ \pm q} \sqrt{\frac{\omega_{0}(q)}{2 \Omega(q)}+\frac{1}{2}}+b_{\mp q}^{+} \sqrt{\frac{\omega_{0}(q)}{2 \Omega(q)}-\frac{1}{2}},
$$

which diagonalize the interacting Hamiltonian as

$$
H(\tau)=E_{\mathrm{GS}}+\sum_{q>0} \Omega(q)\left(d_{q}^{+} d_{q}+d_{-q}^{+} d_{-q}\right)
$$

where $E_{\mathrm{GS}}=\sum_{q>0}\left[\Omega(q)-\omega_{0}(q)\right]$ is the ground-state energy and $\Omega(q)=\sqrt{\omega_{0}(q)^{2}-g(q)^{2}}$ is the spectrum of the elementary excitations.

The dynamics during the quantum quench may be described by the time-dependent annihilation operators as

$$
\begin{aligned}
b_{q}(t) & =u_{q}(t) b_{q}+v_{q}^{*}(t) b_{-q}^{+}, \\
b_{-q}(t) & =u_{q}(t) b_{-q}+v_{q}^{*}(t) b_{q}^{+}
\end{aligned}
$$

where the coefficients obey

$$
i \hbar \partial_{t}\left[\begin{array}{l}
u_{q}(t) \\
v_{q}(t)
\end{array}\right]=\left[\begin{array}{cc}
\omega_{0}(q) & Q(t) g(q) \\
-Q(t) g(q) & -\omega_{0}(q)
\end{array}\right]\left[\begin{array}{l}
u_{q}(t) \\
v_{q}(t)
\end{array}\right]
$$

with the initial conditions $u_{q}(0)=1$ and $v_{q}(0)=0$. At any time instant $\left|u_{q}(t)\right|^{2}-\left|v_{q}(t)\right|^{2}=1$ holds true.

By means of the $u_{q}(t)$ and $v_{q}(t)$ coefficients, the timedependent wave function is expressed as

$$
|\Psi(t)\rangle=\prod_{q>0}\left[\frac{1}{u_{q}^{*}(t)} \exp \left(i \omega_{0}(q) t+\frac{v_{q}^{*}(t)}{u_{q}^{*}(t)} b_{q}^{+} b_{-q}^{+}\right)\right]|0\rangle
$$

where $|0\rangle$ is the initial ground state of the noninteracting system [58]. The wave function depends on the protocol function $Q(t)$ through the coefficients $u_{q}(t)$ and $v_{q}(t)$.

In the present paper, our main goal is to study the optimal $Q(t)$ protocol function with finite duration $\tau$ which results in a final state $|\Psi(\tau)\rangle$ closest to the ground state of the interacting Hamiltonian $H(\tau)$. We investigate two different quantities which both represent a measure of how far the final state is from the interacting ground state. One of them is the expectation value of the total energy in the final state $E_{f}=\langle\Psi(\tau)|H(\tau)| \Psi(\tau)\rangle$. The other quantity is the overlap between the time evolved final state and the ground state of the interacting system $P_{\mathrm{GS}}=|\langle\mathrm{GS} \mid \Psi(\tau)\rangle|^{2}$. In other words, $P_{\mathrm{GS}}$ is the transition probability from the noninteracting to the interacting vacuum. Note that this quantity has been considered numerically in Ref. [11] as the measure for optimization in a related problem.

For generic quench protocol, the energy functional is obtained by calculating the expectation value of Eq. (6) as

$$
E_{f}[Q]=E_{\mathrm{GS}}+\sum_{q>0} 2 \Omega(q) n_{q}[Q]
$$

where the occupation number is the expectation value of the boson numbers in the $+q$ or $-q$ channel. The occupation 
number

$$
\begin{aligned}
n_{q}[Q]= & \left\langle d_{ \pm q}^{+} d_{ \pm q}\right\rangle=\frac{\omega_{0}(q)}{2 \Omega(q)}\left[\left|u_{q}(\tau)\right|^{2}+\left|v_{q}(\tau)\right|^{2}\right] \\
& +\frac{g(q)}{2 \Omega(q)}\left[u_{q}(\tau)^{*} v_{q}(\tau)+u_{q}(\tau) v_{q}(\tau)^{*}\right]-\frac{1}{2}
\end{aligned}
$$

depends on the protocol function through the coefficients $u_{q}(t)$ and $v_{q}(t)$.

The vacuum-to-vacuum probability is obtained by taking the overlap of Eq. (9) with the ground state of the interacting system. Interestingly, the probability depends on the protocol function again through the occupation number only as

$$
\ln P_{\mathrm{GS}}[Q]=-\sum_{q>0} \ln \left(1+n_{q}[Q]\right)
$$

The functionals $E_{f}[Q]$ and $P_{\mathrm{GS}}[Q]$ are highly nonlinear in the protocol function and finding the optimum for arbitrary interaction strength is very complicated using analytic methods. Therefore, the following discussion is restricted to the limiting case of weak interactions. To leading order in the perturbation theory, i.e., when $g_{2} \ll v$ holds, the occupation number is given by

$$
n_{q}[Q]=\frac{g(q)^{2}}{4 \omega_{0}(q)^{2}}\left|\int_{0}^{\tau} d t Q^{\prime}(t) e^{2 i \omega_{0}(q) t}\right|^{2}
$$

where $Q^{\prime}(t)$ is the derivative of the quench protocol. It can be shown that even if forward scattering $\left(g_{4}\right)$ were considered in the interacting Hamiltonian the leading term in Eq. (13) would not depend on $g_{4}$. We substitute Eq. (13) into Eqs. (10) and (12) and keep terms to leading order in the perturbation. In the thermodynamic limit, the summation over the wave numbers turns into an integral leading to

$$
\begin{aligned}
\varepsilon_{f}[Q] & =\frac{E_{f}[Q]-E_{\mathrm{GS}}}{\left|E_{\mathrm{GS}}\right|} \\
& =\int_{0}^{\tau} d t \int_{0}^{\tau} d t^{\prime} Q^{\prime}(t) Q^{\prime}\left(t^{\prime}\right) \frac{\tau_{0}^{2}\left[\tau_{0}^{2}-\left(t-t^{\prime}\right)^{2}\right]}{\left[\tau_{0}^{2}+\left(t-t^{\prime}\right)^{2}\right]^{2}}
\end{aligned}
$$

with the ground-state energy of

$$
E_{\mathrm{GS}}=-\frac{L}{16 \pi v \tau_{0}}\left(\frac{g_{2}}{v}\right)^{2} \frac{1}{\tau_{0}}
$$

and

$$
\begin{aligned}
\mathcal{F}[Q] & =\frac{\ln P_{\mathrm{GS}}[Q]}{\left|E_{\mathrm{GS}}\right| \tau_{0}} \\
& =-\int_{0}^{\tau} d t \int_{0}^{\tau} d t^{\prime} Q^{\prime}(t) Q^{\prime}\left(t^{\prime}\right) \frac{\tau_{0}^{2}}{\tau_{0}^{2}+\left(t-t^{\prime}\right)^{2}}
\end{aligned}
$$

where the dimensionless and nonextensive quantities of $\varepsilon_{f}$ and $\mathcal{F}$ have been introduced. In Eq. (15), $L$ is the length of the system which is considered to be infinitely large in the thermodynamic limit.

We note that the formulas in Eqs. (14) and (16) are valid to leading order of the perturbation theory which is maintained as long as $n_{q}[Q]$ is small in all momentum modes.

\section{PARITY OF THE OPTIMAL QUENCH PROTOCOL}

An important feature of the optimal quench protocol is its symmetries, e.g., the parity. If the protocol is known to have a symmetry, this could reduce significantly the (numerical) effort in determining the optimal ramp.

In Eqs. (14) and (16), we observe that functionals depend on the derivative of the protocol function. Let us split up the derivative of the protocol function into even and odd parts as

$$
Q^{\prime}(t)=p(t)=p_{a}(t)+p_{s}(t)
$$

where $p_{a}(t)=\left[Q^{\prime}(t)-Q^{\prime}(\tau-t)\right] / 2$ is the antisymmetric part while $p_{s}(t)=\left[Q^{\prime}(t)+Q^{\prime}(\tau-t)\right] / 2$ is the symmetric part. The boundary conditions of the protocol function demand

$$
\int_{0}^{\tau} p_{s}(t) d t=1
$$

but the antisymmetric part can be an arbitrary, odd function since its integral vanishes on $[0, \tau]$.

In both the energy functional and the vacuum-to-vacuum transition probability, the kernel of the integral is symmetric under $t \rightarrow \tau-t$ and $t^{\prime} \rightarrow \tau-t^{\prime}$, i.e., when both time variables are reflected. Therefore, the integral part of the functionals is rewritten as

$$
\begin{aligned}
& \int_{0}^{\tau} d t \int_{0}^{\tau} d t^{\prime} p_{s}(t) p_{s}\left(t^{\prime}\right) K\left(t-t^{\prime}\right) \\
& \quad+\int_{0}^{\tau} d t \int_{0}^{\tau} d t^{\prime} p_{a}(t) p_{a}\left(t^{\prime}\right) K\left(t-t^{\prime}\right)
\end{aligned}
$$

and the cross terms proportional to the integral of $p_{s}(t) p_{a}\left(t^{\prime}\right)$ vanish. $K(t)$ is the kernel of Eq. (14) or Eq. (16).

The kernel of the integral is positive (negative) definite for the final energy $\varepsilon_{f}$ (transition probability $\mathcal{F}$ ). This is because the total energy is bounded from below by the ground-state energy and the probability $P_{\mathrm{GS}}$ is bounded from above by 1 . In principle, the boundedness would allow semidefinite kernels but it can be proven by means of Fourier transformation that the kernels of (14) and (16) have no zero eigenvalue on the space of functions with finite duration.

As a consequence, the kernels are positive (negative) definite and so are they on the subspaces of even and odd functions separately. Therefore, the second term of (19) is minimized (maximized) by $p_{a}(t)=0$. In the first term, the symmetric part cannot be chosen as an identically zero function because it would not satisfy the boundary condition Eq. (18). For the antisymmetric part, however, no such condition is prescribed.

Thus, $p_{a}(t)=0$ minimizes the second integral in Eq. (19), which means that the optimal $Q^{\prime}(t)$ function must be an even function, i.e., symmetric under the reflection of $t \rightarrow \tau-t$. Consequently, $Q(t)=1-Q(\tau-t)$ for the optimal quench. In the following sections, protocol functions with this symmetry property will be considered only.

\section{OPTIMAL QUENCH MINIMIZING THE FINAL ENERGY}

This section focuses on minimizing the final energy $\varepsilon_{f}[Q]$ as defined in Eq. (14). 

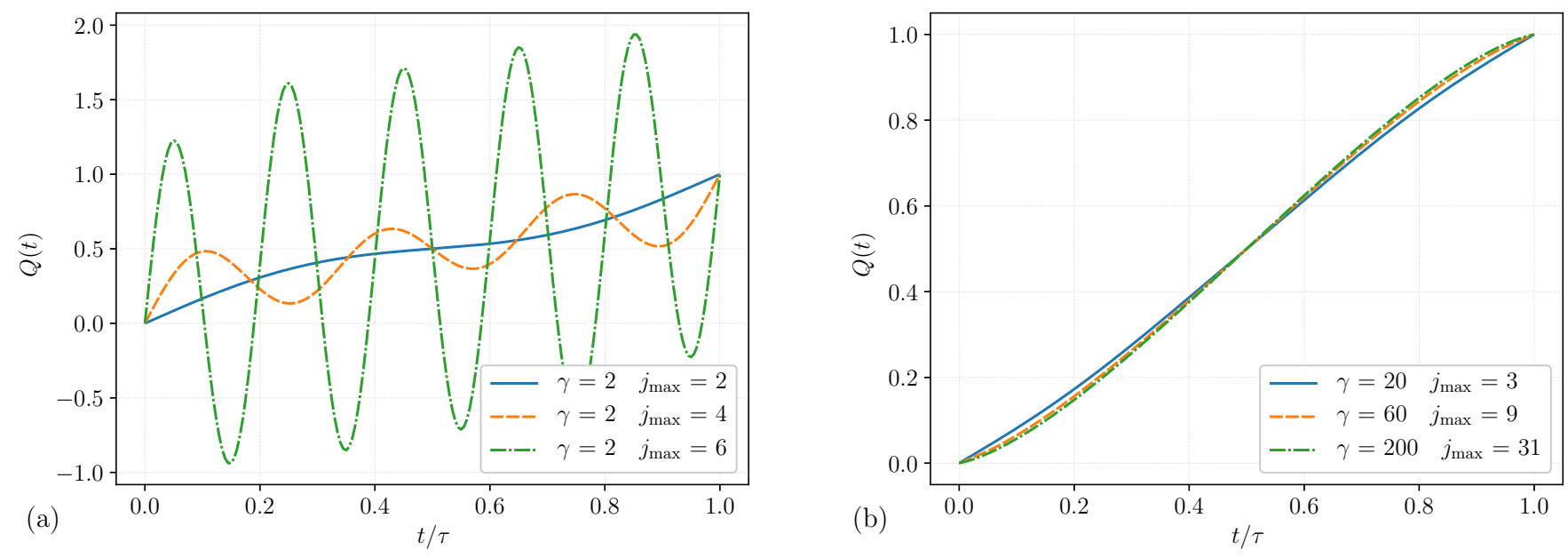

FIG. 1. (a) The optimal quench for $\gamma=2$ with different values of $j_{\max }$, the number of Fourier modes retained. Increasing the truncation index $j_{\max }$ leads to more oscillating behavior of the optimal quench. (b) The optimal quench minimizing the final energy for different values of $\gamma$ and with $j_{\max } \approx \gamma /(2 \pi)$.

Let us consider the Fourier expansion of $Q^{\prime}(t)$ as

$$
Q^{\prime}(t)=\sum_{j=0}^{\infty} \frac{a_{j}}{\tau} \cos \left(\omega_{j} t\right)
$$

where the frequencies $\omega_{j}=2 \pi j / \tau$ have been introduced. Note that the Fourier expansion does not involve any sine function since even functions are considered only in accordance with Sec. III. By using the Fourier expansion, our goal is to find the optimal coefficients $a_{j}$. The final energy functional is obtained as

$$
\varepsilon_{f}[Q]=\sum_{j, j^{\prime}=0}^{\infty} a_{j} M_{j j^{\prime}} a_{j}^{\prime}
$$

where the matrix elements of $\mathbf{M}$ are defined as

$$
\begin{aligned}
M_{j j^{\prime}}= & \frac{1}{\gamma^{2}} \int_{0}^{1} d x \int_{0}^{1} d x^{\prime} \cos (2 \pi j x) \cos \left(2 \pi j^{\prime} x^{\prime}\right) \\
& \times \frac{\frac{1}{\gamma^{2}}-\left(x-x^{\prime}\right)^{2}}{\left(\frac{1}{\gamma^{2}}+\left(x-x^{\prime}\right)^{2}\right)^{2}}
\end{aligned}
$$

with

$$
\gamma=\tau / \tau_{0}
$$

being the dimensionless quench duration. For the $a_{0}$ coefficient, $a_{0}=1$ must hold, which ensures that the integral of $Q^{\prime}(t)$ is 1 . This condition and the minimization of Eq. (21) result in the optimal coefficients of

$$
a_{\mathrm{opt}, j}=\varepsilon_{\min } \cdot\left(\mathbf{M}^{-1}\right)_{j 1}
$$

where $\varepsilon_{\min }=1 /\left(\mathbf{M}^{-1}\right)_{11}$ is the minimal energy.

The matrix elements of $M_{j j^{\prime}}$ cannot be expressed in a closed form for any $j$ and $j^{\prime}$. Therefore, numerical integration is applied. For the numerical calculation, the Fourier series is truncated at $j_{\max }$, i.e., only Fourier components from $j=0$ to $j=j_{\max }-1$ are allowed. Then, the matrix $\mathbf{M}$ has the size of $j_{\max } \times j_{\max }$. In the simulation, the optimal coefficients are computed based on Eq. (24) and the optimal protocol function is reconstructed based on Eq. (20).
Let us first study shorter quenches, for example, $\gamma=2$. The numerically computed optimal quench is shown in Fig. 1(a) for different values of $j_{\max }$. As the truncation index $j_{\max }$ increases, the optimal quench exhibits oscillations with larger and larger amplitude. If further Fourier components are allowed in the quench protocol, the optimal protocol function becomes even more oscillating with even larger amplitudes. These high-frequency components with large amplitude excite bosons far beyond the cutoff energy $1 / \tau_{0}$. In this regime, however, the linear spectrum of the Luttinger model does not apply anymore and, hence, the highly oscillating optimal quench is the consequence of unphysical effects.

In order to stay inside the validity of the Luttinger model, we allow Fourier components with frequencies up to the cutoff energy, i.e., $\omega_{j} \lesssim 1 / \tau_{0}$. In terms of $j$ indices, $j \lesssim \gamma /(2 \pi)$ must hold, which means that $j_{\max }$ should be chosen around $\gamma /(2 \pi)$. This also implies that quenches shorter than $2 \pi \tau_{0}$ inevitably generate excitations in the high-energy regime and, hence, are beyond the validity of the Luttinger model independently from the quench protocol function.

Figure 1(b) shows optimal quench protocol functions in which the truncation index $j_{\max }$ is chosen as the integer part of $\gamma /(2 \pi)$. With this truncation, the optimal protocols are found to be nonoscillating, smooth functions.

Numerical results indicate that the optimal protocol function converges when the quench duration reaches the range of $100 \tau_{0}$. In this regime, it is also observed in the simulation that increasing $j_{\max }$ does not effect the optimal quench protocol, nor does it lead to oscillations. It is an interesting question, how the limiting protocol function can be expressed analytically.

The long quench limit of the functional in Eq. (14) is calculated as

$$
\varepsilon_{f}[Q]=\left\{\left[\tau Q^{\prime}(0)\right]^{2}+\left[\tau Q^{\prime}(\tau)\right]^{2}\right\} \frac{\ln \gamma}{\gamma^{2}}+O\left(\gamma^{-2}\right)
$$

if the protocol function is an analytic function of time. Note that $Q^{\prime}$ scales with $\tau^{-1}$, therefore the leading term is proportional to $\tau^{-2} \ln \left(\tau / \tau_{0}\right)$. Interestingly, the leading term of the 
energy functional depends on the derivative of the protocol function evaluated only at the edges of the quench interval. To minimize the leading term in Eq. (25), the optimal protocol function must fulfill

$$
Q^{\prime}(0)=Q^{\prime}(\tau)=0
$$

for long quenches. The exact characteristics of $Q(t)$ are then chosen in such a way that next-to-leading corrections are minimized.

Since this problem is complicated using analytical methods, numerical method is applied. During the simulation, it is found that for a long quench duration the optimal Fourier coefficients obey power-law behavior as

$$
a_{\mathrm{opt}, j}= \begin{cases}1 & \text { if } j=0 \\ -\frac{A}{j^{\beta}} & \text { if } j \geqslant 1\end{cases}
$$

where $A$ and $\beta$ are numeric parameters. The power-law behavior is also expected for long quenches when the energy scales $1 / \tau$ and $1 / \tau_{0}$ are widely separated. Between these scales, there is a wide energy range in which no dominant energy scale is present and, hence, the $a_{j}$ coefficients are expected to obey a scale-free $j$ dependence.

Applying the condition of (26) to the protocol function with power-law Fourier components described in Eq. (27),

$$
Q^{\prime}(0)=\frac{1}{\tau}\left(1-\sum_{j=1}^{\infty} \frac{A}{j^{\beta}}\right)=0 \Longrightarrow A=\frac{1}{\zeta(\beta)}
$$

where $\zeta$ is the Riemann zeta function. Therefore, the optimal protocol function reads as

$$
\begin{aligned}
Q(t) & =\frac{t}{\tau}-\frac{1}{2 \pi \zeta(\beta)} \sum_{j=1}^{\infty} \frac{\sin \left(\omega_{j} t\right)}{j^{\beta+1}} \\
& =\frac{t}{\tau}-\frac{1}{2 \pi \zeta(\beta)} \operatorname{Im}\left\{\operatorname{Li}_{\beta+1}\left(e^{i 2 \pi \frac{t}{\tau}}\right)\right\}
\end{aligned}
$$

with $\operatorname{Li}_{v}(x)$ being the polylogarithm function. The value of the $\beta$ parameter must be set in such a way that the next-toleading term in the energy functional proportional to $\gamma^{-2}$ is minimized. The optimal value cannot be obtained analytically but must be handled numerically. We performed simulations with durations up to $\tau / \tau_{0}=10000$ and where the truncation index ranges from 10 to 50 . The $\beta$ parameter is obtained by nonlinear curve fit on the optimal $a_{j}$ coefficients.

Based on the numerical study, the optimal quench of a long duration has the form of Eq. (29) with approximately

$$
\beta \approx 1.4 \text {. }
$$

The minimal energy is approximately $\varepsilon_{\min } \approx 8.0 \gamma^{-2}$ and

$$
E_{\min }=E_{\mathrm{GS}}+8.0\left(\frac{g_{2}}{v}\right)^{2} \frac{L}{16 \pi v \tau^{2}} .
$$

The second term measures the energy amount which is inevitably present in the form of excitations after a quench of finite length. Interestingly, this term is independent from the cutoff $1 / \tau_{0}$ and is, therefore, universal for one-dimensional systems within perturbation theory. The final energy as a function of the quench duration is plotted in Fig. 3.
Finally, we note that for short times the optimal quench protocol behaves as a power-law function with the exponent of $\beta$ as

$$
Q(t \ll \tau) \approx \frac{\sin \left(\frac{\beta \pi}{2}\right) \Gamma(-\beta)}{2 \pi \zeta(\beta)}\left(2 \pi \frac{t}{\tau}\right)^{\beta}
$$

where $\Gamma(x)$ is the gamma function.

\section{OPTIMAL QUENCH MAXIMIZING THE VACUUM-TO-VACUUM TRANSITION PROBABILITY}

In this section, the optimal quench maximizing the overlap between the final state and the interacting ground state as defined in Eq. (16) is studied. Similarly to the final energy, the Fourier series of $Q^{\prime}(t)$ is considered as given in Eq. (20). Numerical results imply that Fourier components with frequencies above the cutoff $1 / \tau_{0}$ result in unphysical oscillations for shorter quenches. In order to stay within the validity of the Luttinger model, Fourier components above the cutoff should be omitted and, therefore, the truncation index $j_{\max }$ is chosen as the integer part of $\gamma /(2 \pi)$ for the numerical simulation.

Numerical results are shown in Fig. 2. These indicate that the optimal quench tends to be linear for longer quenches. In the case of $\tau \gg \tau_{0}$, the optimal quench can be derived analytically. First, the functional $\mathcal{F}$ is rewritten as

$$
\mathcal{F}[Q]=-\frac{\pi}{\gamma} \int_{0}^{\tau} d t \int_{0}^{\tau} d t^{\prime} Q^{\prime}(t) Q^{\prime}\left(t^{\prime}\right) \delta_{\gamma}\left(\frac{t-t^{\prime}}{\tau}\right)
$$

where

$$
\delta_{\gamma}(x)=\frac{1}{\pi} \frac{\frac{1}{\gamma}}{\frac{1}{\gamma^{2}}+x^{2}}
$$

has been introduced. In the limit of long quench, $\lim _{\gamma \rightarrow \infty} \delta_{\gamma}(x)=\delta(x)$ is the Dirac-delta function and the functional is obtained as

$$
\mathcal{F}[Q]=-\frac{\pi}{\gamma} \int_{0}^{\tau} d t \tau\left[Q^{\prime}(t)\right]^{2} .
$$

This functional is maximized by the linear quench

$$
Q(t)=\frac{t}{\tau}
$$

Interestingly, this optimal, linear quench is the $\beta \rightarrow 1$ limit of the optimal quench for the minimal energy given in Eq. (29). The maximal probability is calculated as $\mathcal{F}_{\max }=-\pi / \gamma$ and, hence,

$$
\ln P_{\mathrm{GS}, \max }=-\left(\frac{g_{2}}{v}\right)^{2} \frac{L}{16 v \tau},
$$

which is also a universal value since it is independent from the cutoff, $\tau_{0}^{-1}$. Note that Eq. (37) describes the maximal probability of finding the final state in the interacting ground state if a quantum quench of finite duration is applied. The maximal transition probability as a function of the quench duration is plotted in Fig. 3. 

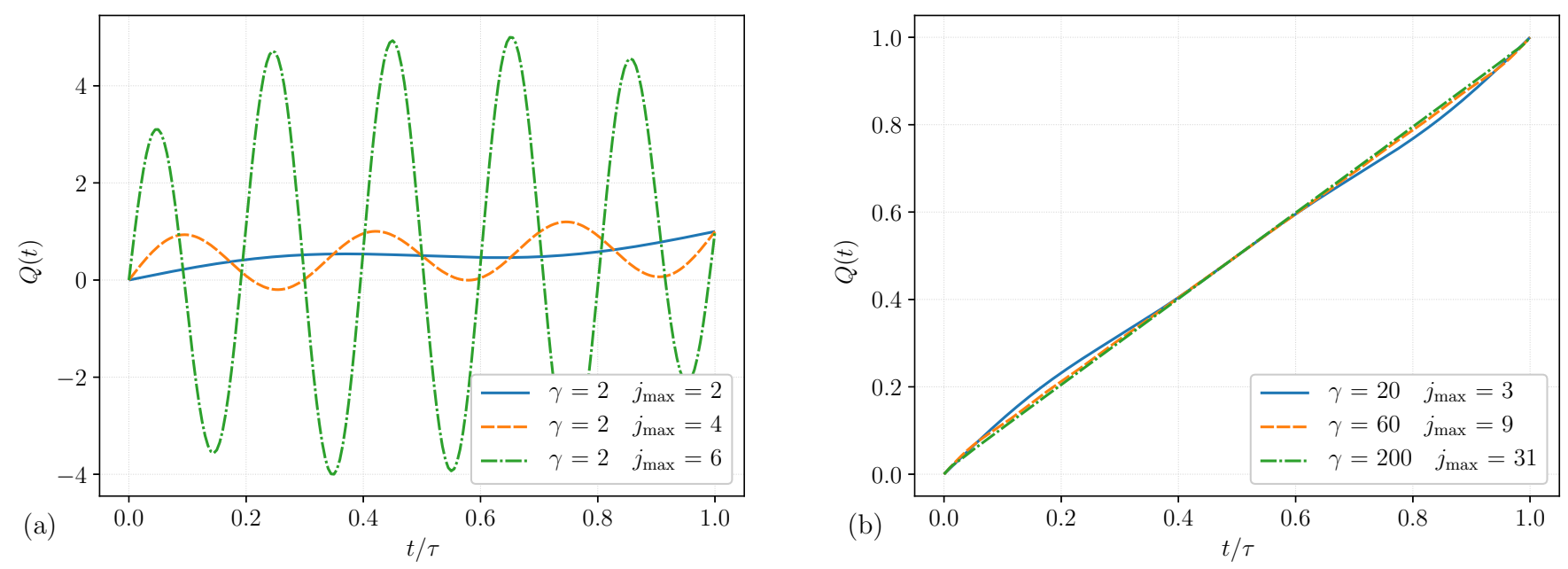

FIG. 2. (a) The optimal quench for $\gamma=2$ with different values of the truncation index $j_{\max }$. For larger $j_{\max }$, the optimal quench has more oscillations. (b) The optimal quench maximizing the vacuum-to-vacuum transition probability for different values of $\gamma$ and with $j_{\max } \approx \gamma /(2 \pi)$.

\section{SUMMARY AND DISCUSSION}

In this paper, we studied the nonequilibrium behavior of the Luttinger model under quenches of finite rate. The low-energy bosonic Hamiltonian in Eq. (1) depends on time through the protocol function $Q(t)$ which switches on a weak interaction. We optimized the $Q(t)$ so as to get the system as close to the ground state of the final Hamiltonian as possible by the end of the quench. Two measures of deviation from the target state were used for this purpose: the excess energy at the end of the quench and the overlap between the time evolved final wave function and the interacting ground state.

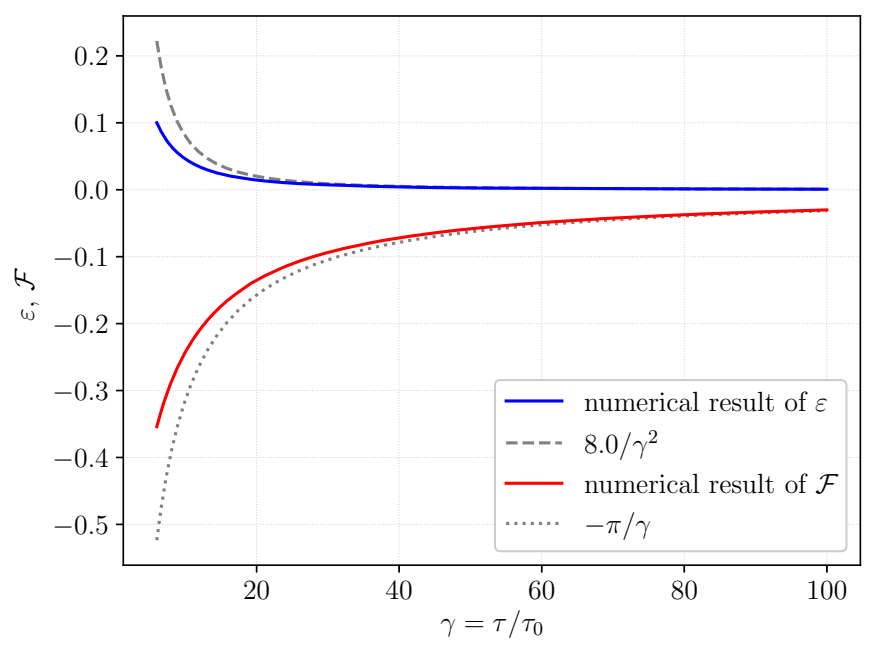

FIG. 3. The minimal energy as a function of quench duration obtained by numerical simulation (blue/solid). For long quenches, the simulation results converge to Eq. (31) (gray/dashed). The logarithm of the vacuum-to-vacuum transition probability as a function of quench duration obtained by numerical simulation (red/solid). For long quenches, the simulation results converge to Eq. (37) (gray/dotted).
We have shown that the optimal protocol must be symmetric with respect to the midpoint of the quench duration. For short quenches $\tau \lesssim \tau_{0}$, the optimal quench exhibits sharp oscillations which are related to bosons excited to very high energies and are beyond the realm of the effective lowenergy model. To avoid these excitations, longer quenches with $\tau \gg \tau_{0}$ are considered within the validity range of the Luttinger model. In this case, the optimal quenches do not exhibit wild oscillation and the protocol functions are found in closed forms in Eqs. (29) and (36) for the case of weak final interactions. The optimal protocols are shown in Fig. 4.

For these ramp protocols, the minimal energy and the maximal vacuum-to-vacuum probability are expressed in Eqs. (31) and (37). These values are independent of the cutoff and are therefore universal within the perturbation theory. These

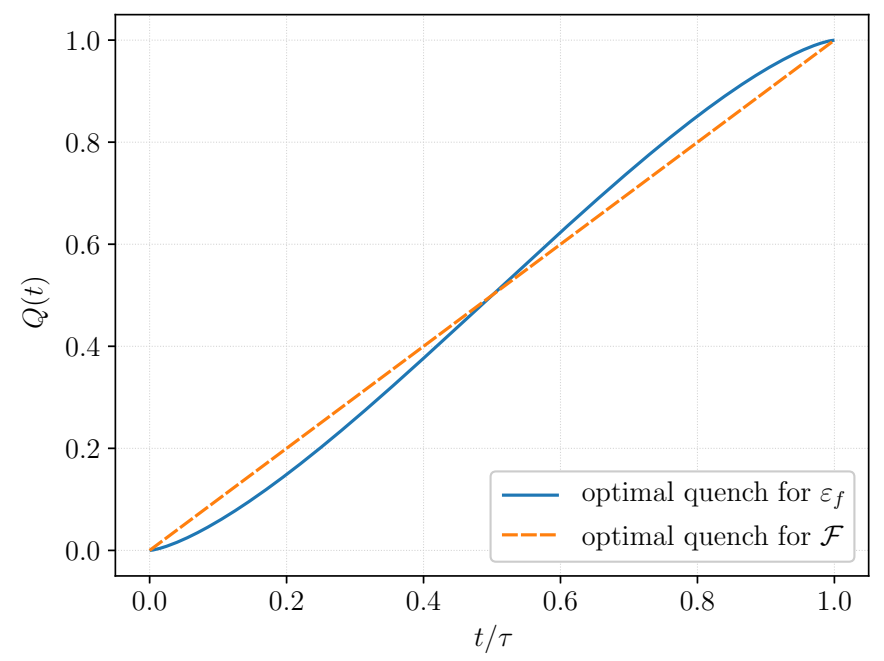

FIG. 4. The optimal protocol function which minimizes the final energy (blue/solid) from Eq. (29), and which maximizes the vacuum-to-vacuum probability (orange/dashed). The latter has a simple linear time dependence. 
analytical protocol functions, shown in Fig. 4, are optimal in the thermodynamic limit. It remains to be investigated to what extent these protocols remain valid beyond the realm of weak interactions.

Our approach of expanding in a Fourier series up to a physically motivated cutoff is different in spirit from finding numerically exact optimum paths by large-scale numerics (e.g., as done in Refs. [11,18]), or from finding mathematically exact optimal protocols for systems having a simpler description (e.g., as done in Refs. [4,15]). One could also expand $Q(t)$ in a power series; we have found that the same main results [oscillatory $Q(t)$ for small $\tau$ and different universal curves for minimizing energy and for maximizing overlap] are also found with such an expansion. However, we believe that the Fourier description presented in this paper has a more physical interpretation.

\section{ACKNOWLEDGMENTS}

This research is supported by the National Research, Development and Innovation Office-NKFIH within the Quantum Technology National Excellence Program (Project No. 2017-1.2.1-NKP-2017-00001) Grants No. K119442 and No. SNN118028, by a BME-Nanonotechnology FIKP grant of EMMI (BME FIKP-NAT), and by the Romanian National Authority for Scientific Research and Innovation, UEFISCDI, Project No. PN-III-P4-ID-PCE-2016-0032.
[1] T. Kadowaki and H. Nishimori, Phys. Rev. E 58, 5355 (1998).

[2] J. Brooke, D. Bitko, T. F., Rosenbaum, and G. Aeppli, Science 284, 779 (1999).

[3] E. Farhi, J. Goldstone, S. Gutmann, and M. Sipser, arXiv:quant$\mathrm{ph} / 0001106$ (2000).

[4] X. Chen, A. Ruschhaupt, S. Schmidt, A. del Campo, D. Guéry-Odelin, and J. G. Muga, Phys. Rev. Lett. 104, 063002 (2010).

[5] E. Torrontegui, S. Ibáñez, X. Chen, A. Ruschhaupt, D. Guéry-Odelin, and J. G. Muga, Phys. Rev. A 83, 013415 (2011).

[6] A. del Campo, Phys. Rev. A 84, 031606(R) (2011).

[7] A. del Campo, Phys. Rev. Lett. 111, 100502 (2013).

[8] R. Modak, L. Vidmar, and M. Rigol, Phys. Rev. E 96, 042155 (2017).

[9] S. Deng, P. Diao, Q. Yu, A. del Campo, and H. Wu, Phys. Rev. A 97, 013628 (2018).

[10] X. Li, D. Pecak, T. Sowiński, J. Sherson, and A. E. B. Nielsen, Phys. Rev. A 97, 033602 (2018).

[11] A. Rahmani and C. Chamon, Phys. Rev. Lett. 107, 016402 (2011).

[12] T. Karzig, A. Rahmani, F. von Oppen, and G. Refael, Phys. Rev. B 91, 201404(R) (2015).

[13] A. Rahmani, B. Seradjeh, and M. Franz, Phys. Rev. B 96, 075158 (2017).

[14] T. Caneva, T. Calarco, R. Fazio, G. E. Santoro, and S. Montangero, Phys. Rev. A 84, 012312 (2011).

[15] K. Takahashi, Phys. Rev. E 87, 062117 (2013).

[16] S. Campbell, G. De Chiara, M. Paternostro, G. M. Palma, and R. Fazio, Phys. Rev. Lett. 114, 177206 (2015).

[17] M. V. Berry, J. Phys. A 42, 365303 (2009).

[18] T. Caneva, M. Murphy, T. Calarco, R. Fazio, S. Montangero, V. Giovannetti, and G. E. Santoro, Phys. Rev. Lett. 103, 240501 (2009).

[19] A. del Campo, M. M. Rams, and W. H. Zurek, Phys. Rev. Lett. 109, 115703 (2012).

[20] A. B. Özgüler, R. Joynt, and M. G. Vavilov, Phys. Rev. A 98, 062311 (2018).

[21] R. Barankov and A. Polkovnikov, Phys. Rev. Lett. 101, 076801 (2008).

[22] A. del Campo and K. Sengupta, Eur. Phys. J.: Spec. Top. 224, 189 (2015).
[23] T. Giamarchi, Quantum Physics in One Dimension (Oxford University, Oxford, 2004).

[24] A. O. Gogolin, A. A. Nersesyan, and A. M. Tsvelik, Bosonization and Strongly Correlated Systems (Cambridge University, Cambridge, England, 1998).

[25] M. A. Cazalilla, R. Citro, T. Giamarchi, E. Orignac, and M. Rigol, Rev. Mod. Phys. 83, 1405 (2011).

[26] K. Schönhammer, J. Phys.: Condens. Matter 25, 014001 (2012).

[27] M. A. Cazalilla, Phys. Rev. Lett. 97, 156403 (2006).

[28] G. S. Uhrig, Phys. Rev. A 80, 061602(R) (2009).

[29] A. Iucci and M. A. Cazalilla, Phys. Rev. A 80, 063619 (2009).

[30] P. Barmettler, M. Punk, V. Gritsev, E. Demler, and E. Altman, New J. Phys. 12, 055017 (2010).

[31] A. Mitra and T. Giamarchi, Phys. Rev. Lett. 107, 150602 (2011).

[32] A. Mitra, Phys. Rev. Lett. 109, 260601 (2012).

[33] C. Karrasch, J. Rentrop, D. Schuricht, and V. Meden, Phys. Rev. Lett. 109, 126406 (2012).

[34] J. Rentrop, D. Schuricht, and V. Meden, New J. Phys. 14, 075001 (2012).

[35] D. M. Kennes and V. Meden, Phys. Rev. B 88, 165131 (2013).

[36] A. Mitra, Phys. Rev. B 87, 205109 (2013).

[37] S. Ngo Dinh, D. A. Bagrets, and A. D. Mirlin, Phys. Rev. B 88, 245405 (2013).

[38] E. Coira, F. Becca, and A. Parola, Eur. Phys. J. B 86, 55 (2013).

[39] D. M. Kennes, C. Klöckner, and V. Meden, Phys. Rev. Lett. 113, 116401 (2014).

[40] M. Schiró and A. Mitra, Phys. Rev. Lett. 112, 246401 (2014).

[41] M. A. Cazalilla and M.-C. Chung, J. Stat. Mech.: Theor. Exp. (2016) 064004.

[42] A. Calzona, F. M. Gambetta, M. Carrega, F. Cavaliere, T. L. Schmidt, and M. Sassetti, SciPost Phys. 4, 23 (2018).

[43] D. B. Gutman, Y. Gefen, and A. D. Mirlin, Phys. Rev. B 81, 085436 (2010).

[44] J. Lancaster and A. Mitra, Phys. Rev. E 81, 061134 (2010).

[45] E. Perfetto, G. Stefanucci, and M. Cini, Phys. Rev. Lett. 105, 156802 (2010).

[46] I. V. Protopopov, D. B. Gutman, P. Schmitteckert, and A. D. Mirlin, Phys. Rev. B 87, 045112 (2013).

[47] M. Schiró and A. Mitra, Phys. Rev. B 91, 235126 (2015). 
[48] E. Langmann, J. L. Lebowitz, V. Mastropietro, and P. Moosavi, Phys. Rev. B 95, 235142 (2017).

[49] J. Dubail, J.-M. Stéphan, and P. Calabrese, SciPost Phys. 3, 019 (2017).

[50] B. Dóra, M. Haque, and G. Zaránd, Phys. Rev. Lett. 106, 156406 (2011).

[51] J. Dziarmaga and M. Tylutki, Phys. Rev. B 84, 214522 (2011).

[52] E. Perfetto and G. Stefanucci, Europhysics Letters 95, 10006 (2011).

[53] F. Pollmann, M. Haque, and B. Dóra, Phys. Rev. B 87, 041109(R) (2013).
[54] R. Sachdeva, T. Nag, A. Agarwal, and A. Dutta, Phys. Rev. B 90, 045421 (2014).

[55] J.-S. Bernier, R. Citro, C. Kollath, and E. Orignac, Phys. Rev. Lett. 112, 065301 (2014).

[56] P. Chudzinski and D. Schuricht, Phys. Rev. B 94, 075129 (2016).

[57] S. Porta, F. M. Gambetta, F. Cavaliere, N. Traverso Ziani, and M. Sassetti, Phys. Rev. B 94, 085122 (2016).

[58] B. Dóra, F. Pollmann, J. Fortágh, and G. Zaránd, Phys. Rev. Lett. 111, 046402 (2013). 